\title{
Interactive effects in memory for harmonic intervals
}

\author{
DIANA DEUTSCH \\ Center for Human Information Processing \\ University of California at San Diego, La Jolla, California 92093
}

\begin{abstract}
Subjects compared the pitches of two temporally separated tones, which were both accompanied by tones of lower pitch. The standard (S) and comparison (C) tones were either identical in pitch or they differed by a semitone. However, the tone accompanying the $S$ tone was always identical in pitch to the tone accompanying the $\mathrm{C}$ tone. Thus, when the $\mathrm{S}$ and $\mathrm{C}$ tones were identical, the intervals formed by the $\mathrm{S}$ and $\mathrm{C}$ combinations were also identical. When the $\mathrm{S}$ and $\mathrm{C}$ tones differed, the intervals formed by the $\mathrm{S}$ and $\mathrm{C}$ combinations also differed. The $\mathrm{S}$ and $\mathrm{C}$ tones were separated by a retention interval during which six extra tones were interpolated. The tones in the second and fourth serial positions of the interpolated sequence were also accompanied by tones of lower pitch. It was found that when the intervals formed by the interpolated (I) combinations were identical in size to the interval formed by the $S$ combination, errors in pitch recognition judgment were fewer than when the sizes of the intervals formed by the I combinations were chosen at random. When the intervals formed by the I combinations differed in size by a semitone from the interval formed by the $\mathrm{S}$ combination, errors in pitch recognition were more numerous than when the sizes of the intervals formed by the I combinations were chosen at random.
\end{abstract}

When two tones are presented simultaneously or in succession, there results the perception of a musical interval; and intervals whose component tones are separated by the same frequency ratio are perceived as being the same in size. That is, tone pairs $F_{1}$ and $F_{2}$, and pairs $F_{3}$ and $F_{4}$, form intervals which are perceptually equivalent when $F_{1} / F_{2}=F_{3} / F_{4}$. This perceptual equivalence of musical intervals is so strong that it forms part of the basis of traditional music theory. The musical scale is based on the semitone, which represents a frequency ratio of approximately 18:17. There are 12 semitones to the octave, and tone pairs within the octave which are separated by the same number of semitones are given the same name (Table 1). When a harmonic or melodic sequence is transposed, so that the same abstract sequence is presented in a different pitch range, the intervals between simultaneous or successive tones remain invariant (Attneave \& Olson, 1971; Deutsch, 1969).

Deutsch (1969) suggested that abstracted interval information is place-coded by the nervous system; and that such coding is achieved through a projection of lower-order onto higher-order units, in a fashion analogous to that proposed by Hubel and Wiesel (1962) for tilt detectors in the visual system. It was further proposed (Deutsch, 1975a) that memory for such abstracted information is based on a continuum whose

This work was supported by United States Public Health Service Grant MH-21001. Requests for reprints should be sent to Diana Deutsch, Department of Psychology, C-009, University of California at San Diego, La Jolla, California 90293. elements are activated by the simultaneous or successive presentation of pairs of tones. Tone pairs which stand in the same ratio project onto the same elements, and therefore onto the same point along the continuum; tone pairs standing in closely similar ratios project onto adjacent points, and so on. It was further proposed that the interactive effects that take place along this continuum are analogous to those occurring within the system that retains absolute pitch values. Such effects include consolidation through repetition (Deutsch, 1972a, 1975b) and similarity-based interference (Deutsch, 1972b, 1973, 1975a, 1975c).

The present experiment was designed to test this hypothesis. Earlier informal studies had indicated that subjects who had not been trained to attach verbal labels to musical intervals were somewhat confused by the task of judging whether such intervals were the same or different in size. Yet, when such subjects made recognition judgments concerning the absolute pitches of tones, these judgments were found to be heavily influenced by the relational context in which the test tones were placed (Deutsch \& Roll, 1974). That is, the subjects tended to judge two tones as identical in pitch when they occurred in the context of harmonic intervals of identical size; and as different when they occurred in the context of harmonic intervals of different sizes. So it was decided to exploit this effect in order to examine whether memory for harmonic intervals was subject to the same types of interactive effect as memory for absolute pitch values.

The basic paradigm was as follows. Subjects compared the pitches of two temporally separated tones, 
Table 1

The Interval Size Continuum

\begin{tabular}{lcccccccccccc}
$\begin{array}{l}\text { Approximate } \\
\text { Ratio }\end{array}$ & 1.06 & 1.12 & 1.19 & 1.26 & 1.33 & 1.41 & 1.50 & 1.59 & 1.68 & 1.78 & 1.89 & 2.00 \\
$\begin{array}{l}\text { Number of } \\
\text { Semitones }\end{array}$ & 1 & 2 & 3 & 4 & 5 & 6 & 7 & 8 & 9 & 10 & 11 & 12 \\
$\begin{array}{l}\text { Musical } \\
\text { Interval }\end{array}$ & $\begin{array}{c}\text { Minor } \\
\text { 2nd }\end{array}$ & $\begin{array}{c}\text { Major } \\
\text { 2nd }\end{array}$ & $\begin{array}{c}\text { Minor } \\
\text { 3rd }\end{array}$ & $\begin{array}{c}\text { Major } \\
\text { 3rd }\end{array}$ & $\begin{array}{c}\text { Perfect } \\
\text { 4th }\end{array}$ & $\begin{array}{c}\text { Diminished } \\
\text { 5th }\end{array}$ & $\begin{array}{c}\text { Perfect } \\
\text { 5th }\end{array}$ & $\begin{array}{c}\text { Minor } \\
\text { 6th }\end{array}$ & $\begin{array}{c}\text { Major } \\
\text { 6th }\end{array}$ & $\begin{array}{c}\text { Minor } \\
\text { 7th }\end{array}$ & $\begin{array}{c}\text { Major } \\
\text { 7th }\end{array}$ & Octave \\
\hline
\end{tabular}

Note-The continuum is here divided into semitonal steps for purposes of clarity, but these steps simply represent arbitrary increments along the continuum.

which were both accompanied by tones of lower pitch. The standard (S) and comparison (C) tones were either identical in pitch or they differed by a semitone. However, the tone accompanying the $S$ tone was always identical in pitch to the tone accompanying the $\mathrm{C}$ tone. Thus, when the $S$ and $C$ tones were identical in pitch, the intervals formed by the $\mathrm{S}$ and $\mathrm{C}$ combinations were also identical in size. When the $\mathrm{S}$ and $\mathrm{C}$ tones differed in pitch, the intervals formed by the $S$ and $C$ combinations also differed in size. The $\mathrm{S}$ and $\mathrm{C}$ tones were separated by a retention interval during which six extra tones were interpolated. The tones in the second and fourth serial positions of the interpolated sequence were also accompanied by tones of lower pitch. In one set of conditions, the intervals formed by the interpolated (I) combinations were identical to the interval formed by the $S$ combination. In a second set, the intervals formed by the I combinations were a semitone larger or smaller than the interval formed by the S combination. In a third set of conditions, the intervals formed by the I combinations were no greater than an octave but were chosen at random from outside this range.

It was expected, following Deutsch and Roll (1974), that subjects would base their pitch recognition judgments in part on their recognition of a sameness or difference in the intervals formed by the $S$ and $C$ combinations. It was further expected that the I combinations would affect memory for the interval formed by the $S$ combination, in the same way as I tones affect memory for the pitch of an S tone (Deutsch, 1972a, $1972 b, 1973,1975 a, 1975 b, 1975 c)$. So it was expected that, in consequence, pitch recognition judgments would vary systematically as a function of the relationship between the intervals formed by the I combinations and the interval formed by the $\mathrm{S}$ combination. That is, an enhancement in recognition performance was expected, to result from interpolating intervals of identical size to that formed by the S combination. And a decrement in performance was expected to result from interpolating intervals that were a semitone larger or smaller in size than that formed by the $\mathrm{S}$ combination.

\section{METHOD}

\section{Procedure}

Subjects listened to an $\mathrm{S}$ tone, which was followed by a se- quence of six interpolated tones, and then, after a pause, by a $\mathrm{C}$ tone. The $\mathrm{S}$ and $\mathrm{C}$ tones were always accompanied by tones of lower pitch, as were the tones in the second and fourth serial positions of the interpolated sequence. The subjects were instructed to listen to the upper tone of the $\mathrm{S}$ combination and ignore the lower tone, to ignore all the intervening tones, and then to judge whether the upper tone of the $C$ combination was the same or different in pitch from the upper tone of the $S$ combination. They indicated their judgments by writing " $\mathrm{S}$ " (same) or "D" (different) on paper.

\section{Temporal Parameters}

All tones were $300 \mathrm{msec}$ in duration and separated by 500 -msec pauses, except that a 2 -sec pause intervened between the last interpolated tone and the $C$ combination. Sequences were presented in groups of 12 , with $10-\mathrm{sec}$ pauses between sequences within a group and 2 -min pauses between groups.

\section{Conditions}

Throughout the experiment, the $\mathrm{S}$ and $\mathrm{C}$ tones were either identical in pitch or they differed by a semitone. However, the tone accompanying the $S$ tone was always identical to the tone accompanying the $\mathrm{C}$ tone. Thus, when the $\mathrm{S}$ and $\mathrm{C}$ tones were identical, the intervals formed by the $S$ and $C$ combinations were also identical. When the $S$ and $C$ tones differed, the intervals formed by the $\mathrm{S}$ and $\mathrm{C}$ combinations also differed in size by a semitone.

A summary of the different conditions of the experiment, together with their names, is included in Table 2. There were three conditions in which the $S$ and $C$ tones were identical in pitch, so that the intervals formed by the $S$ and $C$ combinations were also identical in size. In Condition S1, the I combinations formed intervals that were identical in size to the interval formed by the $\mathrm{S}$ combination. In Condition $\mathrm{S} 2$, the $I$ combinations formed intervals that were a semitone larger or smaller than the interval formed by the $\mathrm{S}$ combination. On half of these sequences, the I combinations formed intervals that were a semitone larger, and on half they were a semitone smaller. In Condition S3, the intervals formed by the I combinations were chosen at random from within an octave, except that intervals were excluded which were identical in size to the interval formed by the $S$ combination or that were a semitone larger or smaller. Intervals were also excluded that were inversions of the interval formed by the $S$ combination or of intervals that were a semitone larger or smaller than that formed by the $\mathrm{S}$ combination. For each sequence, the sizes of the intervals formed by the two I combinations were identical.

There were four conditions in which the $\mathrm{S}$ and $\mathrm{C}$ tones differed in pitch, so that the intervals formed by the $S$ and $C$ combinations differed in size. In Condition D1, the I combinations formed intervals that were identical in size to the interval formed by the $S$ combination. In Condition $D 2$, the I combinations formed intervals that differed in size by a semitone from the interval formed by the $S$ combination. Here, whenever the interval formed by the $\mathrm{C}$ combination was a semitone larger than the interval formed by the $S$ combination, the intervals 
Table 2

Percent Average Error in the Different Conditions of the Experiment

\begin{tabular}{|c|c|c|c|}
\hline . & $\begin{array}{c}\text { Sizes of Intervals Formed } \\
\text { by S and I Combinations } \\
\text { Identical }\end{array}$ & $\begin{array}{c}\text { Sizes of Intervals Formed } \\
\text { by } S \text { and I Combinations } \\
\text { Differ by a Semitone }\end{array}$ & $\begin{array}{c}\text { Sizes of Intervals Formed } \\
\text { by I Combinations } \\
\text { Chosen at Random }\end{array}$ \\
\hline \multirow{2}{*}{$\begin{array}{l}\text { S and C Tones } \\
\text { Identical }\end{array}$} & \multirow[b]{2}{*}{9.7} & \multirow[b]{2}{*}{13.9} & \multirow[b]{2}{*}{11.3} \\
\hline & & & \\
\hline$S$ and $C$ Tones & D1 & D2 $\mathrm{II}$ & D3 \\
\hline Different & 11.4 & 18.3 & 15.3 \\
\hline Total & 10.6 & 16.4 & 13.3 \\
\hline
\end{tabular}

formed by the I combinations were a semitone smaller. Whenever the interval formed by the $\mathrm{C}$ combination was a semitone smaller, the intervals formed by the I combinations were a semitone larger. Thus there was no relationship of identity between the interval formed by the $\mathrm{C}$ combination and the intervals formed by the I combinations. In Condition $\mathrm{D} 2 \mathrm{II}$, the I combinations also formed intervals that differed in size by a semitone from the interval formed by the $S$ combination. But here, whenever the interval formed by the $C$ combination was a semitone larger than the interval formed by the $S$ combination, the intervals formed by the I combinations were also a semitone larger. Whenever the interval formed by the $\mathrm{C}$ combination was a semitone smaller, the intervals formed by the I combinations were also a semitone smaller. In this condition, therefore, the intervals formed by the I combinations were always identical in size to the interval formed by the $\mathrm{C}$ combination. In Condition D3, the intervals formed by the I combinations were chosen at random from within an octave, except that intervals were excluded which were identical in size to the interval formed by the $S$ combination or that were a semitone larger or smaller. Intervals were also excluded that were inversions of the interval formed by the $\mathrm{S}$ combination, or of intervals that were a semitone larger or smaller than that formed by the $S$ combination. For each sequence, the sizes of the intervals formed by the two I combinations were identical.

There were 12 sequences in each condition, except that Condition S2 contained 24 sequences, in order to match the number of sequences in Conditions $\mathrm{D} 2 \mathrm{I}$ and $\mathrm{D} 2 \mathrm{II}_{\mathrm{II}}$ combined. There were, therefore, 96 sequences altogether, and these were presented in randem order. The subjects listened to the entire set of sequences on 2 separate days, and their results were averaged.

\section{$S$ and $C$ Combinations}

The frequencies of the $S$ and $C$ tones were taken from the equal-tempered scale (International Pitch, $A=435 \mathrm{~Hz}$ ), and they ranged over an octave, from the $\mathrm{F}$ above middle $\mathrm{C}$ to the $E$ above. The frequencies employed (in hertz) were: $F=345$, $F \#=366, G=388, G \#=411, A=435, A \#=461, B=488$, $\mathrm{C}=517, \mathrm{C} \#=548, \mathrm{D}=581, \mathrm{D} \#=615$, and $\mathrm{E}=652 . \mathrm{S}$ combinations forming each of the 12 intervals within the octave were employed equally often in all conditions. In the conditions where the $S$ and $C$ tones differed, on half of the sequences the $\mathrm{C}$ tone was a semitone higher than the $\mathrm{S}$ tone; on the other half it was a semitone lower.

\section{Intervening Tones}

The intervening tones were also taken from the equaltempered scale $(A=435 \mathrm{~Hz})$, and ranged from the $A$ below middle $\mathrm{C}$ to the $\mathrm{E}$ over an octave above. The frequencies employed (in hertz) were: $A=218, A \#=230, B=244$, $\mathrm{C}=259, \mathrm{C} \#=274, \mathrm{D}=290, \mathrm{D} \#=308, \mathrm{E}=326, \mathrm{~F}=345$,
$F \#=366, G=388, G \#=411, A=435, A \#=461, B=488$, $\mathrm{C}=517, \mathrm{C} \#=548, \mathrm{D}=581, \mathrm{D} \#=615$, and $\mathrm{E}=652$. The tones were chosen at random from this set, with the following restrictions. No interpolated sequence contained repeated tones or tones that were separated by octaves. Further, no interpolated sequence contained tones that were the same in pitch as the $\mathrm{S}$ or $\mathrm{C}$ tones of that sequence, or their accompanying tones, or that were separated by an octave from any of these. These restrictions also applied to the lower tones of the I combinations.

\section{Apparatus}

Tones were generated by two Wavetek oscillators controlled by a PDP-8 computer, and the output was recorded on highfidelity tape. The tape was played to subjects on a high-quality tape recorder through two loudspeakers. The components of each simultaneous combination were presented through different loudspeakers, and the single tones were presented through both loudspeakers.

\section{Subjects}

Thirty-four undergraduates at the University of California at San Diego served as subjects for this experiment. They were selected on the basis of obtaining a score of at least $80 \%$ correct on a small tape containing sequences designed as in Conditions S3 and D3 (intervals formed by the I combinations chosen at random). The selection ratio was about $1: 5$. The subjects were paid for their services.

\section{RESULTS}

The error rates in the different conditions of the experiment are shown in Table 2. It can be seen that the predicted differences were, in fact, obtained. First, when the I combinations formed intervals of identical size to the interval formed by the $\mathrm{S}$ combination, the error rates were lower than when the intervals formed by the I combinations were chosen at random. This effect was found to be highly significant (Conditions S1 and D1 vs. Conditions S3 and D3, p $<.005$, one-tailed, on a Wilcoxon test).

Second, when the I combinations formed intervals that were a semitone larger or smaller than the interval formed by the $\mathbf{S}$ combination, the error rates were higher than when the intervals formed by the I combinations were chosen at random. This effect was also found to be highly significant (Conditions S2 and D2 I vs. Conditions S3 and D3, p $<.005$, one-tailed, on a 
Wilcoxon test). Condition D2 II was excluded from this analysis, since in this condition the I combinations formed intervals of identical size to the interval formed by the $\mathrm{C}$ combination. It was expected, by analogy with studies on memory for the pitch of a single tone, that this pattern of relationships would give rise to a greater increase in errors (Deutsch, 1973). And, indeed, the error rate in Condition D2 II was found to be even higher than in Condition D2 , where this relationship of identity was not present. The difference between these two conditions did not, however, reach statistical significance.

\section{DISCUSSION}

It is concluded from these findings that memory for harmonic interval information is indeed subject to interactive effects which are analogous to those found in memory for the pitch of a single tone (Deutsch, 1975c). A statistically significant consolidation of abstracted interval information was here demonstrated, as was similarity-based interference in memory for such information.

The finding of similarity-based interference supports the hypothesis that memory for abstracted interval information is the function of a continuum whose elements are activated by the presentation of tone pairs, and that this continuum is arranged in terms of similarity between the frequency ratios formed by these tone pairs. This finding is in accordance with the results of Plomp, Wagenaar, and Mimpen (1973), who required subjects to identify musical intervals produced by simultaneous tones, and found that confusions were made on the basis of interval size (and not, for instance, on the basis of frequency ratio simplicity). In general, the closer the intervals were in size, the larger the number of confusions. These authors conclude that such stimuli are arranged along a continuum that is organized in terms of interval size.

The hypothesized interval size continuum should, however, be qualified to accommodate the phenomenon of inversion. Two chords are considered harmonically equivalent when their component tones are placed in different octaves. Thus a simultaneous tone pair which forms an interval of a semitone is perceptually similar to a tone pair which forms an interval of $12-\mathrm{n}$ semitones. Experimental evidence for the perceptual similarity of inverted intervals has been obtained by Deutsch and Roll (1974) and Plomp, Wagenaar, and Mimpen (1973) and a mechanism to account for this phenomenon has been proposed by Deutsch (1969). Because inversions represent a special case, they were excluded from the design of the present experiment.

The issue may be raised of the possible mediation of verbal labeling in the present results. To examine this, the subjects were asked at the end of the experiment whether they knew how to label musical intervals, and if they had been doing this during the experiment. Six of the 34 subjects replied that they were able to attach labels to intervals to some extent, though none with any confidence, and they all denied having done so during the experiment. Further, the error patterns for these subjects were not discernibly different from those of the others. An explanation in terms of verbal labeling therefore appears most improbable.

This paper has been addressed to the question of memory organization for harmonic intervals as uncovered in a highly controlled setting. However, the issue may be raised of the applicability of these (and other such) findings to normal musical listening situations. In Western traditional music, there are strong a priori anu cransilionai probabilities governing both linear successions of tones and also harmonic sequences (Piston, 1948). This highly overlearned system of probabilities must play an important role in the retrieval of musical information from memory, in the same way as our knowledge of grammatical structure facilitates the retrieval of verbal materials. It has been shown, for instance, that short-term recognition of melodic patterns is superior when these are in our tonal system than when they are not (Francés, 1972; Zenatti, 1969); and it is reasonable to expect that analogous results would be obtained with harmonic sequences also. Thus, in listening to actual music, the interference effects uncovered here may be compensated for by such higher-order processes.

\section{REFERENCES}

Attneave, F., \& Olson, R. K. Pitch as a medium: A new approach to psychological scaling. American Journal of Psychology, 1971, 84, 147-165.

Deutsch, D. Music recognition. Psychological Review, 1969, 76. 300-307.

Deutsch, D. The effect of repetition of standard and comparison tones on recognition memory for pitch. Joumal of Experimental Psychology, 1972, 93, 156-162. (a)

DEUTSCH. D. Mapping of interactions in the pitch memory store. Science, 1972, 175, 1020-1022. (b)

DE UTCH, D. Interference in memory between tones adjacent in the musical scale. Journal of Experimental Psychology, 1973, 100. 228-231.

Deutsch, D. Auditory memory. Canadian Journal of Psychology. 1975, 29. 87.105. (a)

DEUTSCH. D. Facilitation by repetition in recognition memory for tonal pitch. Memory \& Cognition, 1975, 3, 263-266. (b)

DE UTSCH, D. The organization of short-term memory for a single acoustical attribute. In D. Deutsch \& J. A. Deutsch (Eds.), Short-term memory. New York: Academic Press, 1975. (c)

Deutsch, D., \& Roll, P. L. Error patterns in delayed pitch comparison as a function of relational context. Journal of Experimental Psychology, 1974, 103, 1027-1034.

Francés, R. La perception de la musique. Paris: Vrin, 1972.

Hubel, D. H., \& Wiesel, T. N. Receptive fields, binocular interaction and functional architecture in the cat's visual cortex. Journal of Physiology, 1962, 160, 106-154.

Piston, W. Harmony (2nd ed.). London: Norton, 1948.

Plomp, R., Wagenaar, W. A., \& Mimpen, A. M. Musical interval recognition with simultaneous tones. Acustica. 1973, 29. 102-109.

Zenatti, A. Le developement génétique de la perception musicale. Monographies Françaises de Psychologie, 1969, 17, 1-110.

(Received for publication December 6, 1977; revision accepted May 11, 1978.) 